\title{
Sarcoidosis Presenting as Cranial Multinevritis
}

\author{
Sara Xavier Pires ${ }^{\mathrm{a}, \mathrm{c}}$, Guiomar Pinheiro ${ }^{\mathrm{a}}$, Luisa Serpa Pinto ${ }^{\mathrm{a}}$, \\ Teresa Sequeira ${ }^{a}$, Antonio Marinho ${ }^{b}$
}

\begin{abstract}
A 29-year-old woman presented to the emergency department with red eyes and hypoacusia since the previous weeks associated with a sudden right facial palsy. Ophthalmologic examination revealed bilateral uveitis and lumbar puncture was compatible with aseptic meningitis. Thoracic computed tomography scan showed pulmonary nodules and mediastinal and hilar lymphadenopathy. Bronchoalveolar lavage revealed a CD4/CD8 ratio of 8 , and lymph node biopsy confirmed noncaseating granulomas. The diagnosis of sarcoidosis with ocular, pulmonary, and neurologic involvement was made. Due to sarcoidosis severity at presentation, the patient was started on systemic steroids and methotrexate with improvement. Sarcoidosis involvement of the central nervous system is a rare condition $(5-10 \%)$ but can be the first manifestation of the disease. Its presentation can be heterogeneous; therefore, symptoms are frequently devalued by patients and physicians, leading to a late diagnosis and permanent damage. Clinicians' awareness is crucial to early diagnosis and treatment.
\end{abstract}

Keywords: Sarcoidosis; Cranial nerves palsy; Uveitis; Autoimmunity

\section{Introduction}

Sarcoidosis is an inflammatory disease of unknown cause, characterized by the presence of non-caseating granulomas. It shows different local and racial spread patterns. Women appear to be slightly more susceptible than men, and it often occurs in young otherwise healthy adults. At least $5 \%$ of sarcoidosis patients will have a family member with the disease; however, most cases are sporadic [1].

Although sarcoidosis can affect virtually every organ of the body, lung involvement with asymptomatic bilateral hilar and mediastinal lymphadenopathy is the most frequent, af-

Manuscript submitted December 13, 2020, accepted December 22, 2020

Published online January 19, 2021

${ }^{a}$ Internal Medicine Department, Centro Hospitalar e Universitario do Porto, Porto, Portugal

${ }^{\mathrm{b}}$ Clinical Immunology Unit, Centro Hospitalar e Universitario do Porto, Porto, Portugal

${ }^{\mathrm{c} C o r r e s p o n d i n g ~ A u t h o r: ~ S a r a ~ X a v i e r ~ P i r e s, ~ I n t e r n a l ~ M e d i c i n e ~ D e p a r t m e n t, ~}$ Centro Hospitalar e Universitario do Porto, Largo Prof Abel Salazar 4099-001

Porto, Portugal. Email: saraixp@gmail.com

doi: https://doi.org/10.14740/jmc3635 fecting more than $80 \%$ of patients [2], followed by eye with ocular manifestations occurring in $20-30 \%$ of the patients [3], and skin in $25 \%$ of the patients [4]. Central nervous system involvement, reported in $5-10 \%$ of sarcoidosis patients, is less common and its presentation can be heterogeneous. When categorizing the neurologic manifestations of sarcoidosis, it is observed that one-third to one-half of the patients can have more than one type of neurologic presentation [5].

We present a case of sarcoidosis with lung, eye, and neurological involvement. We consider this case relevant because it alerts to the heterogeneity of neurological involvement, which can be the first manifestation of the disease and is sometimes undervalued by the patient. This warning leads to the importance of early treatment when it is adequate to avoid permanent damage. In addition, we aim to describe and discuss in detail our clinical approach in order to increase available data and knowledge about this disease.

\section{Case Report}

The authors present a case of a 29-year-old woman with alopecia (since she was 16 years old), myopia, and familiar history of sarcoidosis (mother), who presented to the emergency department (ED) with sudden onset of right facial paralysis. She occasionally had episodes of uveitis and, in the previous week, noticed a slight redness of the eyes with a burning eye sensation, which worsened in the previous 2 days. She also mentioned having tinnitus and a minor hearing loss in the prior 3 weeks. In the ED, she had no fever and presented hemodynamically stable with normal breath sounds. Neurologic examination revealed intact mental status and balance, as well as normal motor and sensory functions. However, it showed involvement of the VII and VIII nerves with right peripheral facial palsy and decreased left hearing acuity. The remaining cranial nerves were intact.

Initial blood tests showed lymphopenia $\left(0.89 \times 10^{3} / \mu \mathrm{L}\right)$ with otherwise normal blood count and metabolic panel. C-reactive protein level was normal, and the erythrocyte sedimentation rate was slightly elevated $(24 \mathrm{~mm} / \mathrm{h})$. Cranial computed tomography (CT) was normal, and thoracic CT scan showed several small pulmonary nodules (maximum of $7 \mathrm{~mm}$ of diameter) and multiple mediastinal and hilar lymphadenopathies (Fig. 1).

The patient underwent lumbar puncture (LP), and the cerebrospinal fluid (CSF) analysis revealed 14 white blood cells/ $\mu \mathrm{L}$ with a predominance of mononuclear cells $(12$ cells $/ \mu \mathrm{L})$, and normal glucose and proteins levels. CSF's gram stain and culture were unyielding, and nucleic acid amplification as- 

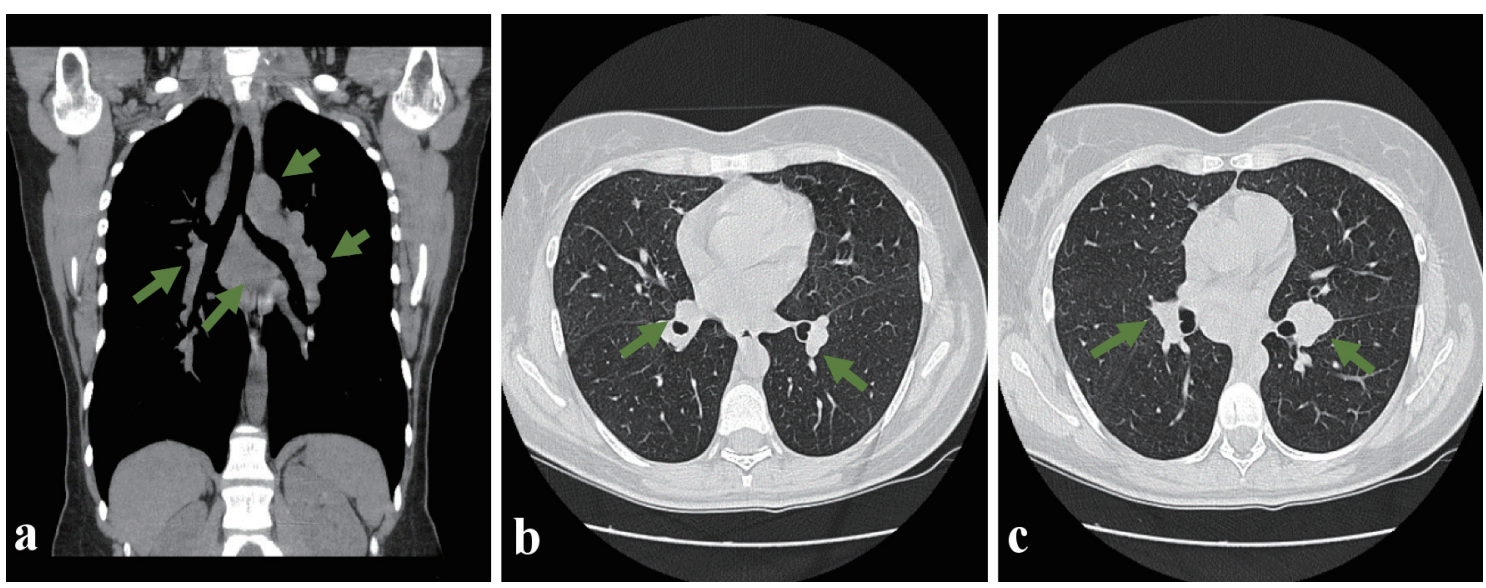

Figure 1. Thoracic CT scan showing multiple mediastinal adenopathies, in all compartments, with the largest ones standing out subcarinal (a), about $37 \mathrm{~mm}$ in diameter, and a $32 \mathrm{~mm}$ pre-tracheal in diameter. (a) Coronal section showing through the green arrows several mediastinal adenopathies; (b, c) Cross-section to show in the pulmonary window the presence of multiple adenopathies and pulmonary nodules (green arrow). CT: computed tomography.

says for cytomegalovirus, enterovirus, herpes simplex (1 and 2), varicella-zoster, herpes 6, and Epstein-Barr virus were all negative. A single immunoglobulin $\mathrm{G}$ (IgG) oligoclonal band was found both in the serum and CSF, with identical characteristics. Serologic and CSF angiotensin-converting enzyme (ACE) levels were normal. Workup for human immunodeficiency virus (HIV) and latent tuberculosis were negative. A presumption diagnosis of sarcoidosis was established. The patient had immediately started corticosteroids (methylprednisolone $500 \mathrm{mg} /$ day for 3 days and then prednisolone $20 \mathrm{mg} /$ day) with a favorable clinical response.

Immunological studies were all negative, including antiphospholipid and antineutrophil cytoplasmic antibodies. Detailed ophthalmic examination revealed bilateral anterior uveitis and right eye papillitis; however, ocular angiography was not compatible with vasculitis.

The patient underwent a bronchoscopy, and bronchoalveolar lavage was obtained, showing a CD4/CD8 ratio of 8 . A lymph node aspirate by endobronchial ultrasound-guided transbronchial needle aspiration was also obtained, revealing the presence of non-caseating granulomas.

The definitive diagnosis of sarcoidosis presenting with lung, ocular and neurologic manifestations, namely aseptic meningitis and multiple cranial nerve involvement, was made 11 weeks after admission to ED.

Afterwards, the prednisolone dose was slowly tapered (12 weeks) until $5 \mathrm{mg} /$ day and then maintained. During this period (6 weeks of treatment), the patient repeated the LP, which revealed normal cytology of the CSF and the thoracic CT scan, which was very similar to the previous one. The audiogram was normal and magnetic resonance imaging (MRI) performed on the 10th day of treatment was also normal.

Due to the severity of its presentation, with neurological involvement, the patient was started on methotrexate $(10 \mathrm{mg} /$ week till a maximum of $12.5 \mathrm{mg} /$ week) and initially maintained a low prednisolone dose ( $5 \mathrm{mg} /$ day), which was subsequently suspended.

After beginning the treatment plan, the patient became asymptomatic, remaining symptom-free even after the corticoid suspension. During 5 years of treatment, the methotrexate dose was also slowly tapered and then suspended, without any sign of symptom relapse. Currently, the patient has remained asymptomatic without any treatment for the last 5 months, remaining under surveillance in ambulatory care.

\section{Discussion}

Sarcoidosis is usually a self-limited, non-threatening disease. It remains mainly a diagnosis of exclusion since there are no definitive diagnostic serological or radiological tests. A biopsy should be obtained from sites most readily accessible such as skin, lymphadenopathies, or lung tissue. The presence of noncaseating granulomas on tissue biopsy and compatible clinical features is usually considered proof of the diagnosis of sarcoidosis. The definitive diagnosis of NS is defined by Stern et al [5] as suggestive clinical presentation after excluding alternative diagnosis and pathologic confirmation of systemic sarcoidosis. Tissue sampling of the nervous system is rarely possible, and given the heterogeneity of the neurologic presentations and the low incidence and prevalence of ocular and NS in general, it has been a challenge to initiate rigorous clinical trials.

The presence of family history raised a high suspicion, and the existence of mediastinal and hilar lymphadenopathies with a bronchoalveolar lavage with a CD4/CD8 ratio of 8 and ultimately the presence of non-caseating granulomas in histology confirmed the sarcoidosis, supporting the initial diagnosis of neurologic and ocular involvement.

Ocular involvement may be asymptomatic, therefore every patient with sarcoidosis should undergo an ophthalmological investigation, including slit-lamp examination, since every part of the eye may be involved, and there is the threat of blindness. Our patient presented with bilateral anterior uveitis and right eye papillitis, without documented optic neuritis, but the ocular angiography was made already under therapy. Optic neuritis can be associated with both anterior and posterior 
uveitis, which usually requires long-term systemic therapy [1].

Neurological involvement is rare, and it usually requires the exclusion of infection, malignancy, vascular and structural brain damage, and other vascular and autoimmune conditions. Certain areas of the nervous system are more commonly affected in NS. These include cranial nerve involvement, aseptic meningitis, hydrocephaly, myelopathy, and anterior hypothalamic disease with associated diabetes insipidus. Peripheral facial nerve palsy (VII cranial nerve) accounts for $50-75 \%$ of all the neurologic presentation of sarcoidosis [6]. It can be transient and mistaken for Bell's palsy (idiopathic seventh nerve paralysis). Because this form of neurosarcoidosis often resolves within weeks and may not recur, it may have occurred before a definitive diagnosis of sarcoidosis. Our patient presented some symptoms compatible with one of the other most severe cranial neuropathies, the involvement of the VIII cranial nerve. It can compromise hearing or vestibular function and can be refractory to treatment, causing functional impairment to the patient [5]. However, she had a normal audiogram, which probably happened because it was made under therapy since the hearing loss was already solved at the exam time.

The presence of granulomatous inflammation can be seen on MRI, and sometimes it can be mistaken for multiple sclerosis. However, the presence of meningeal enhancement or hypothalamic involvement suggests NS. Our patient had a normal MRI, but the CSF analysis revealed lymphocytic meningitis, described in sarcoidosis [5] as well as the presence of oligoclonal bands and an elevated IgG index. CSF ACE level has a poor sensitivity but high specificity for establishing a diagnosis of NS, and it was normal in this case [7].

Sarcoidosis can be considered a benign disease with a good prognosis. It may be treated according to the organs involved, from no treatment to potent and focused immunosuppressive strategies. The presence of organ-threatening diseases, including eye or nervous system involvement, indicates an early treatment to avoid permanent damage. Our patient was started on steroids, and due to the severity of its presentation with confirmed NS, she started a combined therapy with methotrexate. It is the most widely studied cytotoxic agent and it works in approximately two-thirds of sarcoidosis patients, regardless of the disease manifestation [1]. At present, the best way to assess the activity of sarcoidosis is still through clinical activity, and some studies showed that there were no relapses if patients remained asymptomatic for 3 years after prednisolone withdrawal [8].

\section{Acknowledgments}

None to declare.

\section{Financial Disclosure}

The authors did not receive any specific grant from funding agencies in the public, commercial or not-for-profit sectors.

\section{Conflict of Interest}

The authors declare that they have no conflict of interest.

\section{Informed Consent}

Written informed consent was obtained from the patient for publication of this case report and accompanying images.

\section{Author Contributions}

All authors have contributed to the manuscript. Sara Xavier Pires: conceptualization, methodology, writing of original draft, review and editing; Luisa Serpa Pinto and Guiomar Pinheiro: writing of original draft, review and editing; Teresa Sequeira and Antonio Marinho: supervision, visualization, review and editing. All authors read and approved the final version of the manuscript.

\section{Data Availability}

Any inquiries regarding supporting data availability of this study should be directed to the corresponding author.

\section{References}

1. Fauci AS, Kasper DL, Longo DL, Braunwald E, Hauser SL, Jameson JL LJ. Harrison's Principles of Internal Medicine. 19a Edition. In: Longo DL, editor. Boston: McGraw-Hill; 2015.

2. Studdy PR. Oxford Textbook of medicine. 3rd editio. Oxford Medical Publications; 1996. p. 2817-2832.

3. Costabel U. Sarcoidosis: clinical update. Eur Respir J Suppl. 2001;32:56s-68s.

4. Akyol L, Aslan K, Ozgen M, Sayarlioglu M. A rare comorbidity: neurosarcoidosis and cutaneous sarcoidosis. BMJ Case Rep. 2015;1-4.

5. Stern BJ, Aksamit A, Clifford D, Scott TF, Neurosarcoidosis Study G. Neurologic presentations of sarcoidosis. Neurol Clin. 2010;28(1):185-198.

6. Stern BJ, Krumholz A, Johns C, Scott P, Nissim J. Sarcoidosis and its neurological manifestations. Arch Neurol. 1985;42(9):909-917.

7. Saha BK, Saha A, Beegle S. Measurement of cerebrospinal fluid ACE level in aseptic meningitis: diagnostic? BMJ Case Rep. 2019;12(8).

8. Rizzato G, Montemurro L, Colombo P. The late followup of chronic sarcoid patients previously treated with corticosteroids. Sarcoidosis Vasc Diffuse Lung Dis. 1998;15(1):52-58. 